British Journal of Nutrition (2020), 124, 1-13

doi:10.1017/S0007114520000914

(c) The Authors 2020. This is an Open Access article, distributed under the terms of the Creative Commons Attribution licence (http:// creativecommons.org/licenses/by/4.0/), which permits unrestricted re-use, distribution, and reproduction in any medium, provided the original work is properly cited.

\title{
Food groups and the likelihood of non-alcoholic fatty liver disease: a systematic review and meta-analysis
}

\author{
Kaiyin He†, Yuting Li†, Xin Guo, Lu Zhong and Shaohui Tang* \\ Department of Gastroenterology, The First Affiliated Hospital, Jinan University, Guangzhou, Guangdong, People's Republic \\ of China \\ (Submitted 20 September 2019 - Final revision received 4 February 2020 - Accepted 26 February 2020 - First published online 6 March 2020 )
}

\section{Abstract}

Dietary habits have been implicated in the development and severity of non-alcoholic fatty liver disease (NAFLD). Several epidemiological studies attempted to assess the relationship between food groups and the likelihood of NAFLD, but these results were conflicting. The present meta-analysis was conducted to assess the association between food groups and the likelihood of NAFLD. Published literature was retrieved and screened from MEDLINE, Embase and Web of Science. Out of 7892 retrieved articles, twenty-four observational studies (fifteen cross-sectional studies and nine case-control studies) met our eligibility criteria and were finally included in this systematic review and meta-analysis. Consumption of both red meat and soft drinks contributed to a positive association with NAFLD. Inversely, nut consumption was negatively associated with NAFLD. There were no significant influences on the likelihood of NAFLD about consuming whole grains, refined grains, fish, fruits, vegetables, eggs, dairy products and legumes. This meta-analysis suggests that individuals who consumed more red meat and soft drinks may have a significantly increased likelihood of NAFLD, whereas higher nut intake may be negatively associated with NAFLD. Further prospective studies are required to assess the association between food patterns and NAFLD.

Key words: Non-alcoholic fatty liver disease: Liver steatosis: Risk of non-alcoholic fatty liver disease: Diet: Food groups: Metaanalyses: Systematic reviews

With the rising prevalence of obesity, diabetes mellitus and the metabolic syndrome, non-alcoholic fatty liver disease (NAFLD) has been considered the most common liver disease which affects $20-30 \%$ of the worldwide population ${ }^{(1)}$. NAFLD is characterised by the accumulation of hepatic fat $>5 \%$ and not caused by excessive alcohol consumption, use of hepatotoxic medications or other established liver diseases ${ }^{(2)}$. It encompasses a spectrum of liver damage that can progress from simple steatosis to non-alcoholic steatohepatitis, hepatic fibrosis and cirrhosis. Approximately $30 \%$ of patients with simple steatosis progress to non-alcoholic steatohepatitis, which can potentially progress to fibrosis/cirrhosis and eventually lead to hepatocellular carcinoma $^{(3)}$

Metabolic changes, including insulin resistance and impaired lipid metabolism, have been identified as the molecular pathogenesis of NAFLD ${ }^{(4)}$. NAFLD, which is similar to metabolic diseases such as obesity, inflammation, insulin resistance and type 2 diabetes, is considered to be a liver component of the metabolic syndrome ${ }^{(5)}$. The Western dietary pattern characterised by higher loads of energy content, saturated fat, fructose, sugar-sweetened beverages and refined carbohydrates is associated with weight gain, obesity and more recently with NAFLD ${ }^{(6)}$. Although there is currently no consensus on the pharmacological treatment of NAFLD, the international guidelines recommend that lifestyle modification associated with weight loss should be an integral part of the treatment of NAFLD ${ }^{(7)}$.

Lifestyle modifications include achieving weight loss, increasing physical activity and acquiring a healthy dietary pattern ${ }^{(8)}$. Although weight loss is an important approach for the management of NAFLD, extreme dietary intervention for the purposes of weight loss, such as very low-carbohydrate diet, may increase insulin resistance and exacerbate NAFLD even if it can reduce body weight ${ }^{(9)}$. Also, achieving weight loss and maintaining it is often difficult for the most obese patients ${ }^{(10)}$. On the other hand, it has been shown that obese or lean patients with NAFLD benefit more from a healthy diet than from weight reduction $^{(11)}$, suggesting that healthy diet patterns play an important role in the prevention and management of NAFLD. In view of this, several studies have assessed the relationship between these food groups and the likelihood of NAFLD, but results were

\footnotetext{
Abbreviation: NAFLD, non-alcoholic fatty liver disease.

* Corresponding author: Shaohui Tang, email tangshaohui206@163.com

$\dagger$ These authors contributed equally to this work
} 
conflicting $^{(12-18)}$. Thus, to gain a better understanding of the relationship between NAFLD and dietary factors, we searched the literature according to dietary guidelines and guidelines for the prevention and treatment of NAFLD to evaluate the association of the following eleven food groups including refined grains, whole grains, red meat, fish, vegetables, fruits, dairy products, legumes, eggs, nuts and soft drinks, with the likelihood of NAFLD by this meta-analysis ${ }^{(6,19,20)}$.

\section{Method}

This meta-analysis was registered through the International Prospective Register of Systematic Reviews (PROSPERO) as CRD42019120766. This study was reported according to the Meta-analysis Of Observational Studies in Epidemiology (MOOSE) statement and Preferred Reporting Items for Systematic Reviews and Meta-Analyses (PRISMA) ${ }^{(21,22)}$. Similarly, it followed the recommendations of the Cochrane Collaboration Handbook ${ }^{(23)}$. We employed the PICO format (population, intervention, comparison, outcome) to answer the research question: 'Are food groups (refined grains, whole grains, red meat, fish, vegetables, fruits, dairy products, legumes, eggs, nuts and soft drinks) associated with the occurrence of NAFLD?'. Population: adults with NAFLD; intervention: food groups (refined grains, whole grains, red meat, fish, vegetables, fruits, dairy products, legumes, eggs, nuts and soft drinks); comparison: adults without NAFLD; outcome: the occurrence of NAFLD.

\section{Search strategy}

An electronic search was conducted in the MEDLINE, Embase and Web of Science databases with no restrictions to time, language and publication type. Observational studies addressing the association between food groups and NAFLD were eligible. The following search terms were combined to design the search strategy: 'Grain', 'whole grain', 'refined grain', 'cereal', 'coarse cereal', 'meat', 'red meat', 'white meat', 'pork', 'beef', 'poultry', 'domestic fowl', 'fish', 'diary', 'milk', 'yogurt', 'soy', 'legumes', 'natto', 'tofu', 'egg', 'vegetable', 'fruit', 'nut', soft drink', 'carbonate beverage', 'carbonated drinks', 'sugar beverage', 'soda', 'nonalcoholic fatty liver disease', 'NAFLD', 'nonalcoholic steatohepatitis', 'liver steatosis', 'fatty liver', 'hepatic steatosis', 'nutritional profile', 'nutritional intake', 'dietary pattern', 'dietary intake', 'diet', 'nutrition' and 'food'. Duplicate publications were removed.

Our two investigators, K. H. and Y. L., independently screened the studies by title, abstract and full text. When the selected studies were identical, agreement was reached; any disagreement was resolved by consulting the third investigator (S. T.). We also manually searched the additional relevant articles from reference lists of retrieved articles.

\section{Inclusion and exclusion criteria}

Inclusion criteria were as follows: (1) participants: adult participants; (2) observational studies: cohort studies, case-control studies or cross-sectional studies that investigated food groups (whole grains, refined grains, vegetables, fruits, soft drinks, fish, red meat, nuts, milk, eggs and legumes) in relation to the likelihood of NAFLD; (3) diagnosis: NAFLD diagnosis that was determined by ultrasound (diffused echogenicity of the liver or increased echogenicity compared with the renal cortex), or by abdominal computed tomography scan (L:S ratio $\leq 1 \cdot 1$, the L:S ratio was calculated from the mean of the liver and spleen measurements) or by multidetector computed tomography scan (a value of the liver:phantom ratio < $30 \cdot 0$ ) or by MRI (quantified liver fat content) or by proton magnetic resonance spectroscopy (intra-hepatic TAG content more than $5 \%$ ) or compatible liver histology ${ }^{(24-28)}$.

Exclusion criteria: (1) animal studies; (2) adolescents or pregnant women; (3) present of hepatitis B surface antigens, antibody against hepatitis $\mathrm{C}$ or HIV; (4) excess consumption of alcohol (more than $20 \mathrm{~g} / \mathrm{d}$ in women or $30 \mathrm{~g} / \mathrm{d}$ in men) or potentially hepatotoxic drugs (tamoxifen, steroids and amiodarone); (5) other factors which caused hepatic steatosis such as inflammatory bowel disease, coeliac disease or autoimmune hepatitis and (6) diagnosed malignancy.

\section{Data extraction and risk of bias}

Two investigators (K. H. and Y. L.) independently extracted and summarised data from each study. Any discrepancies were resolved by consulting the third investigator (TSH). The quality of the included trials was assessed by the Risk Of Bias In Non-randomized Studies - of Interventions (ROBINS-I) tool ${ }^{(29)}$. It contains seven domains that rank the studies as low, moderate, serious or critical serious. According to the ROBINS-I guidance, if a study is ranked low in all domains, it is considered low risk of bias; if it is ranked low or moderate in all domains, it is considered moderate risk of bias; if it is ranked serious in at least one domain, it is considered serious risk of bias; if it is ranked critical in at least one domain, it is considered critical serious risk of bias $^{(29)}$. All included studies were assessed by two researchers (X. G. and L. Z.), and discrepancies were resolved by consulting the third investigator (S. T.).

\section{Statistical analysis and data synthesis}

We analysed the data using Stata release 15.1 (StataCorp). The results were expressed in terms of OR and $95 \%$ CI. In order to evaluate the weight of each study, the standard error of the logarithmic OR of each study was calculated and taken as the estimated variance of the logarithmic OR. The inverse variance method was adopted ${ }^{(30)}$. Before inclusion in the overall metaanalysis, the results of sex stratification were summarised using the fixed effects model. Different study types (cross-sectional studies or case-control studies or randomised controlled trials or cohort studies) were analysed separately.

Statistical heterogeneity was evaluated by Cochran's Q-test and $I^{2}$ statistics, and $P<0.1$ and $I^{2}>50 \%$ was considered as significant heterogeneity ${ }^{(31)}$. If the heterogeneity was acceptable $\left(I^{2} \leq 50 \%\right)$, a fixed effects model was conducted to calculate the pooled OR. Otherwise, a random effects model was adopted. We used 'metan logor loglb logub, label(namevar=author, yearvar=year) by(study) fixed eform' command to combine studies without significant heterogeneity $\left(I^{2} \leq 50 \%\right)$ and used 'metan logor loglb logub, label(namevar=author, yearvar=year) by(study) random eform' command to combine studies with 


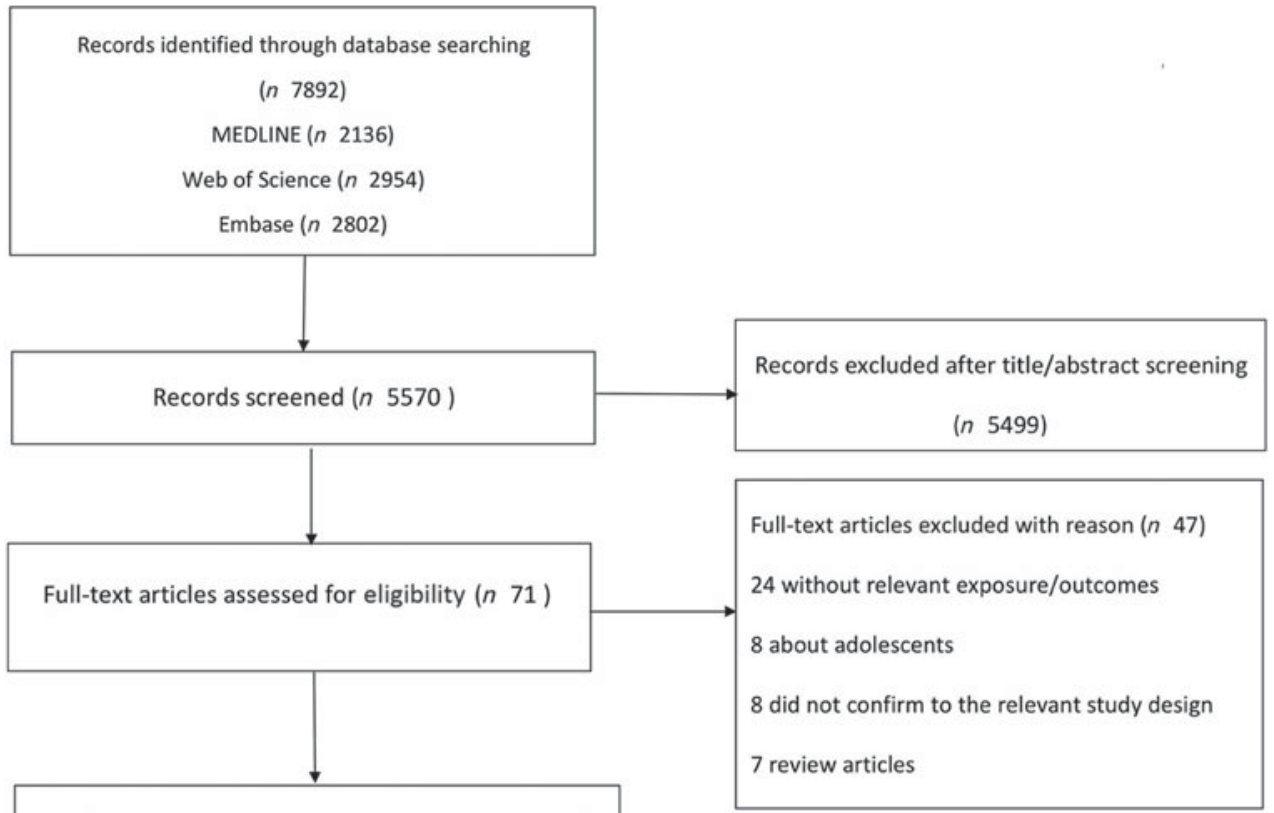

Publications included in the meta-analysis ( $\left.\begin{array}{ll}n & 24\end{array}\right)$

Fig. 1. Flow diagram of literature search and study selection.

significant heterogeneity $\left(I^{2}>50\right)$. If the number of studies is $>5$, the causes of heterogeneity were investigated by subgroup analysis based on geographic location (Asia, Europe and America), number of cases $(\geq 1000 v .<1000)$ and dietary assessment method (validated $v$. non-validated). In addition, sensitivity analysis was performed for low-bias studies (if the number of studies $>5$ ). According to the Cochrane Handbook, if $\geq 10$ studies are available, we explore publication bias by using Egger's tests and funnel plots ${ }^{(32,33)}$

\section{Results}

A total of 7892 potentially relevant articles were identified through literature searching in MEDLINE, Embase and Web of Science, and 2322 duplicate articles were excluded. The remaining 5570 articles undergone a title and abstract screening, and 5499 were further excluded as they did not fulfil the inclusion criteria.

In total, seventy-one articles remained for full-text evaluation. Among them, forty-seven articles were excluded because seven were review articles, eight were about adolescents, twenty-four were without the relevant exposure/outcomes and eight did not conform to the relevant study design. Finally, twenty-four articles were identified and included in this systematic review and meta-analysis ${ }^{(12-15,18,34-52)}$. The study selection process is described in Fig. 1. Among them, fifteen were cross-sectional studies and nine were case-control studies. Of the studies, seventeen studies were conducted in Asia (nine in China, one in South Korea, two in Iran, three in Israel and two in Japan), five in European (three in Greece, two in Italy) and two in America. The main characteristics of the included studies are illustrated in online Supplementary Tables S1 and S2.

\section{Quality assessment}

Fifteen studies were evaluated to have a low risk of bias, and nine studies had a moderate risk of bias. The quality of included studies ranged from low to moderate risk of bias as shown in online Supplementary Table S3. Bias risk of each domain of the included studies is also shown in online Supplementary Table S3.

\section{Red meat}

A total of eight studies assessed the effect of red meat consumption on the likelihood of NAFLD, which include seven crosssectional studies (with 5141 cases) and one case-control study (with 2974 cases) $^{(18,34,36,38,44,48,49,52)}$. Meta-analysis results from the seven homogeneous cross-sectional studies $\left(I^{2}=48.7 \%\right.$, $P=0.069)$ showed a positive association between red meat consumption and the likelihood of NAFLD (OR $=1 \cdot 121 ; 95 \%$ CI 1.042, 1.207; $P=0.002$ ) (Fig. 2). Also, the result from the case-control study found a positive association $(\mathrm{OR}=1 \cdot 150$; $95 \%$ CI 1.023, 1.293; $P=0.020)$.

Further, subgroup analyses were conducted by risk of bias, geographic location, number of cases and dietary assessment. Except for the two studies with $\geq 1000$ cases by which the pooled result showed no association of red meat consumption with the likelihood of NAFLD $\left(I^{2}=0.0 \%, P_{\text {heterogeneity }}=0.340 ; \quad\right.$ OR $=$ $1.005 ; 95 \%$ CI $0.967,1.150 ; P=0.227)$, the results still showed positive associations between red meat consumption and the likelihood of NAFLD in the studies with low risk of bias and $<1000$ cases, and in the studies from the analyses by geographic location and dietary assessment. Evidence of significant heterogeneity in subgroup analysis was only found in the 


Study
ID ES (95\% CI)

Fig. 2. Fixed effects meta-analysis of cross-sectional studies that examined red meat consumption and non-alcoholic fatty liver disease (NAFLD) risk. Weights are from fixed effects analysis. ES, effect size.

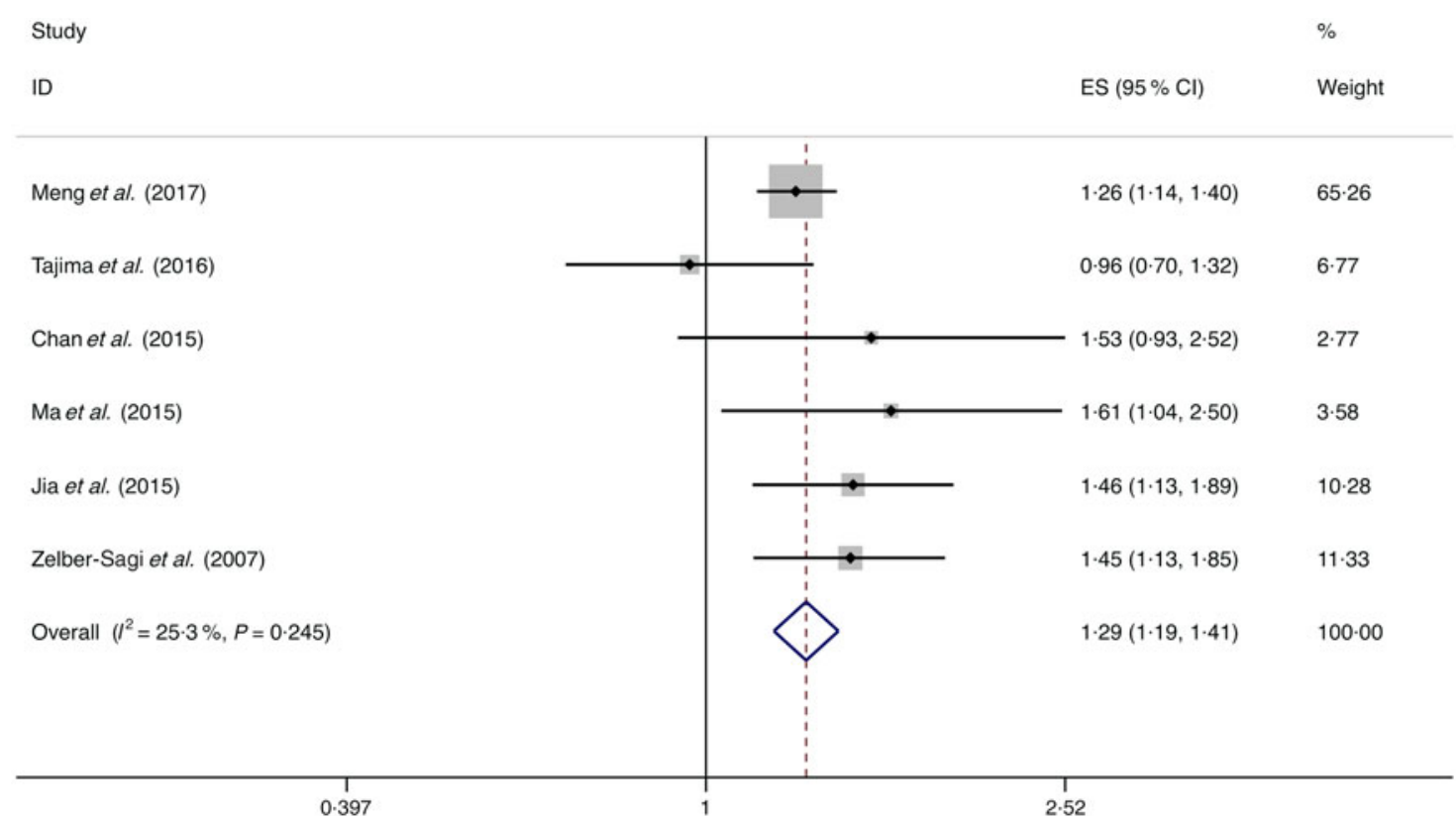

Fig. 3. Fixed effects meta-analysis of cross-sectional studies that examined soft drink consumption and non-alcoholic fatty liver disease (NAFLD) risk. Weights are from fixed effects analysis. ES, effect size.

studies with validated dietary assessment $\left(I^{2}=56 \cdot 4 \%\right.$ ) (online Supplementary Table S4).

\section{Soft drinks}

A total of seven studies assessed the effect of soft drink consumption on the likelihood of NAFLD, which include six cross-sectional studies (with 9887 cases) and one case-control study (with sixty cases) ${ }^{(12,34,38,40,41,46,49)}$. Meta-analysis results from the six homogeneous cross-sectional studies $\left(I^{2}=25 \cdot 3 \%\right.$,
$P=0.245)$ showed that soft drink consumption was positively correlated with the likelihood of NAFLD (OR $=1.294 ; 95 \%$ CI 0.191, 1.406; $P=0.000$ ) (Fig. 3). However, the result from the case-control study found no association between soft drink intake and the possibility of NAFLD (OR $=2 \cdot 000 ; 95 \%$ CI $0 \cdot 894$, 4.472; $P=0.091)$.

Stratified by risk of bias, geographic location, number of cases and dietary assessment in subgroup analyses, the results still indicated positive correlations between beverage intake and the likelihood of NAFLD. Evidence of no significant 
Study

$\%$

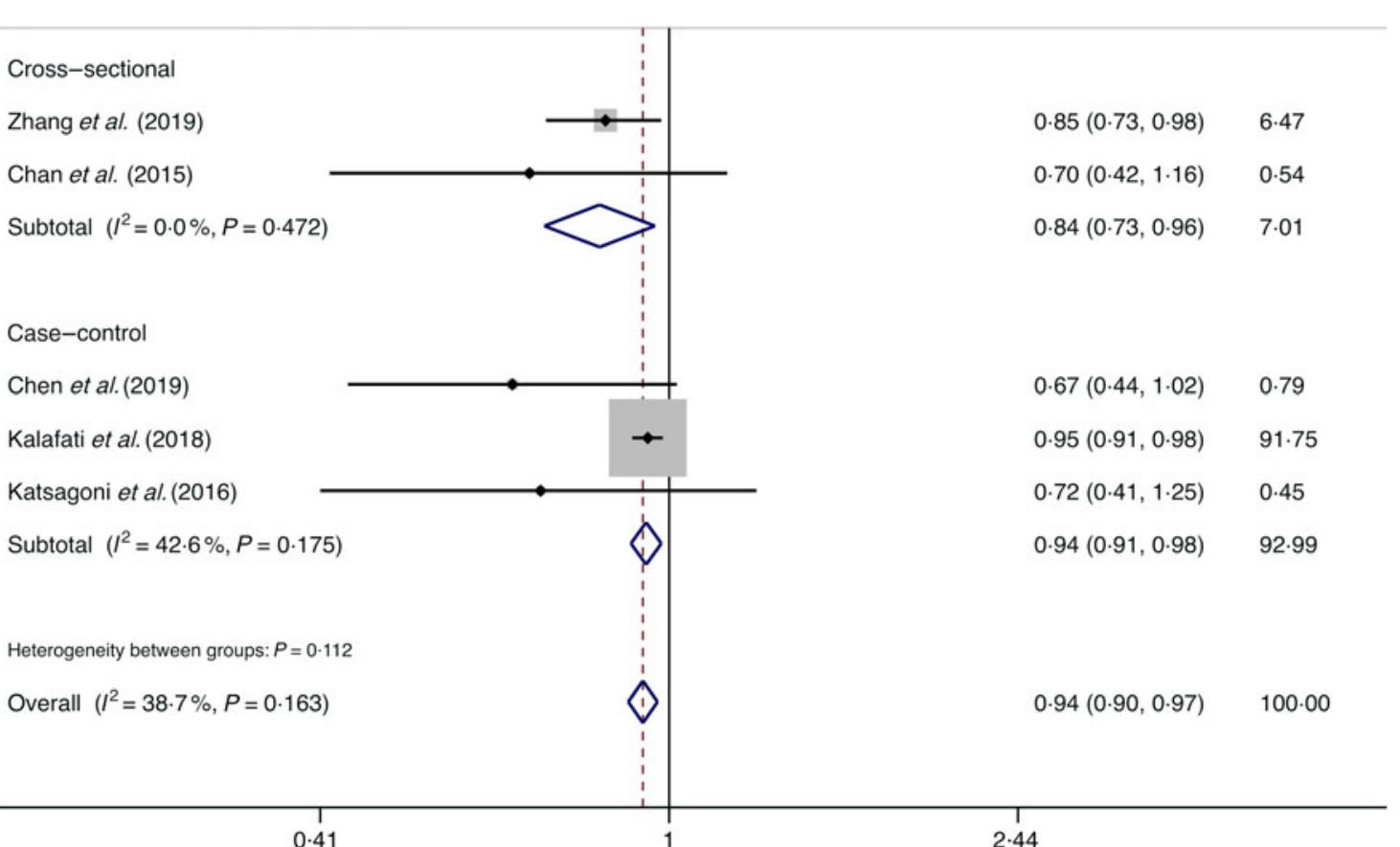

Fig. 4. Fixed effects meta-analysis of prospective studies that examined nut consumption and non-alcoholic fatty liver disease (NAFLD) risk. Weights are from fixed effects analysis. ES, effect size.

heterogeneity was found in subgroup analysis (online Supplementary Table S5).

\section{Nuts}

A total of five studies assessed the effect of nut consumption on the likelihood of NAFLD, which include two cross-sectional studies (with 4737 cases) and three case-control studies (with 768 cases $)^{(15,34,35,39,51)}$. A negative association of nut intake with the possibility of NAFLD was observed among the cross-sectional studies $\left(I^{2}=0.0 \%, P_{\text {heterogeneity }}=0.472 ; \quad\right.$ OR $=0.837$; $95 \%$ CI $0.727,0.965 ; P=0.014)$ and case-control studies $\left(I^{2}=42.6 \%, P_{\text {heterogeneity }}=0.175 ; \quad\right.$ OR $=0.943,95 \%$ CI 0.907 , 0.980; $P=0.003$ ) (Fig. 4).

\section{Whole grains}

A total of three studies assessed the effect of whole-grain consumption on the likelihood of NAFLD, which include two cross-sectional studies (with 2394 cases) and one case-control study (with seventy-three cases) ${ }^{(14,36,37)}$. No significant association between whole-grain consumption and the likelihood of NAFLD was observed among the cross-sectional studies $\left(I^{2}=0.0 \%, \quad P_{\text {heterogeneity }}=0.965 ; \quad\right.$ OR $=0.990 ; \quad 95 \%$ CI 0.965 , $1.015 ; P=0.439)$ and case-control study $(\mathrm{OR}=1.029 ; 95 \% \mathrm{CI}$ $0 \cdot 993,1 \cdot 067 ; P=0 \cdot 119$ ) (Fig. 5).

\section{Refined grains}

A total of six studies assessed the effect of refined grain consumption on the likelihood of NAFLD, which include four cross-sectional studies (with 3509 cases) and two case-control studies (with 207 cases) ${ }^{(34,36-39,46)}$. No significant association between refined grain consumption and the likelihood of NAFLD was observed among the cross-sectional studies $\left(I^{2}=68.4 \%, \quad P_{\text {heterogeneity }}=0.023 ; \quad\right.$ OR $=0.973 ; 95 \%$ CI 0.769 , $1.230 ; \quad P=0.818)$ and case-control study $\left(I^{2}=85.6 \%\right.$, $P_{\text {heterogeneity }}=0.008 ; \quad$ OR $=1.050 ; 95 \%$ CI $0.880,1.253$; $P=0.591)$ (Fig. 6).

\section{Fish}

A total of six studies assessed the effect of fish consumption on the likelihood of NAFLD, which include five cross-sectional studies (with 2780 cases) and one case-control study (with 134 cases $)^{(14,18,34,36,39,49)}$. Meta-analysis results from the five heterogeneous cross-sectional studies $\left(I^{2}=69.4 \%, P=0 \cdot 011\right)$ showed no significant association between fish consumption and the likelihood of NAFLD (OR $=0.908 ; 95 \%$ CI 0.647, 1.276; $P=0.579$ ) (Fig. 7). However, the result from the case-control study showed fish consumption was negatively associated with the possibility of NAFLD (OR $=0.845 ; \quad 95 \%$ CI 0.751 , 0.950; $P=0.005)$.

\section{Fruits}

A total of eight studies assessed the effect of fruit consumption on the likelihood of NAFLD, which include six cross-sectional studies (with 11861 cases) and two case-control studies (with 2168 cases $)^{(14,34,36,38,42,44,45,47)}$. There was no significant association between fruit intake and the likelihood of NAFLD among the cross-sectional studies $\left(I^{2}=68.0 \%, \quad P_{\text {heterogeneity }}=0.008\right.$; $\mathrm{OR}=0.991 ; 95 \%$ CI $0.844,1.163 ; P=0.907)$ (Fig. 8) and 


Baratta et al. (2017)

Fig. 5. Fixed effects meta-analysis of cross-sectional studies that examined nut consumption and non-alcoholic fatty liver disease (NAFLD) risk. Weights are from fixed effects analysis. ES, effect size.

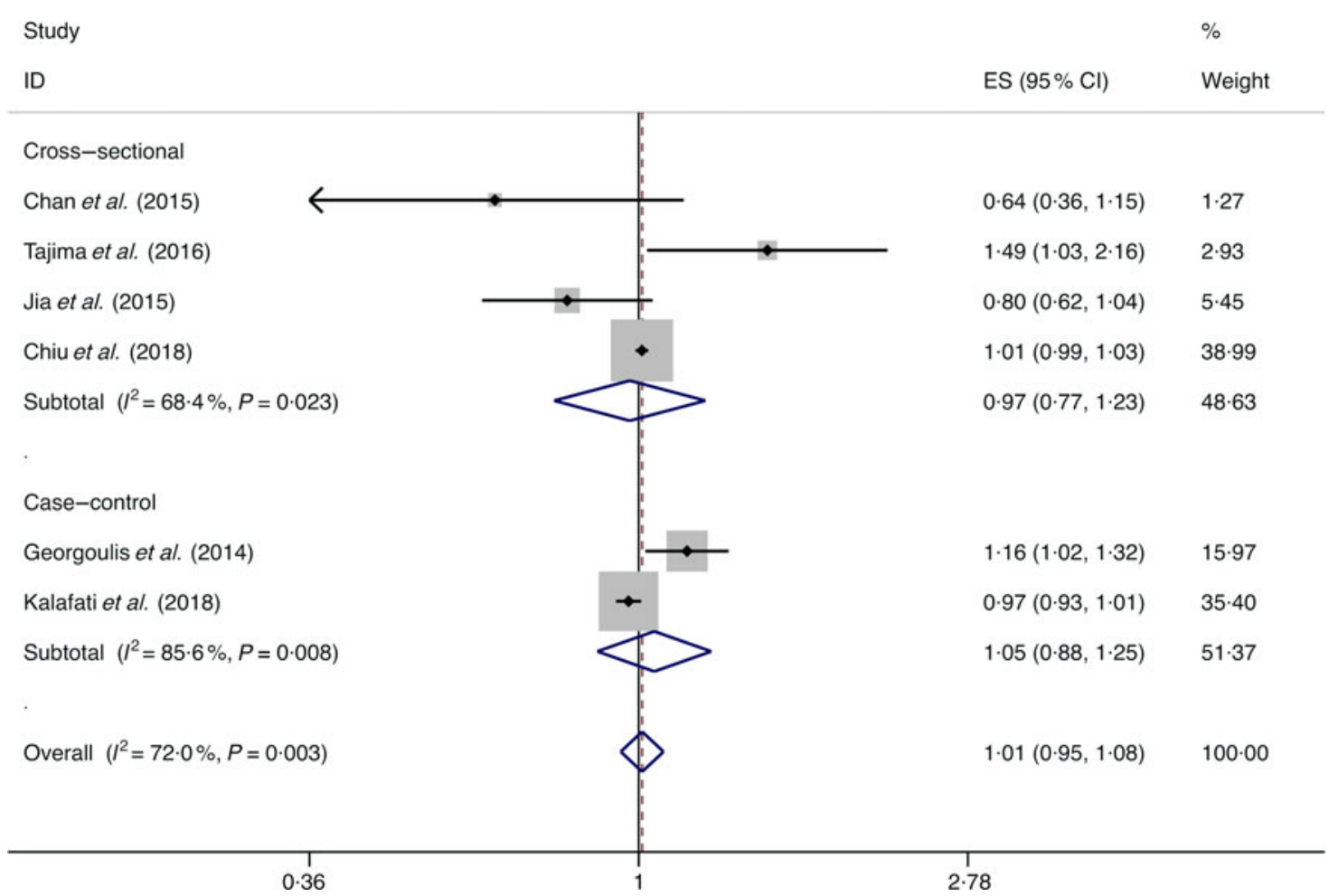

Fig. 6. Random effects meta-analysis of prospective studies that examined refined grain consumption and non-alcoholic fatty liver disease (NAFLD) risk. Weights are from random effects analysis. ES, effect size.

case-control studies $\left(I^{2}=37 \cdot 4 \%, P_{\text {heterogeneity }}=0 \cdot 206 ;\right.$ OR $=0 \cdot 899$; $95 \%$ CI 0.802, 1.007; $P=0.066$ ) (Fig. 9).

In subgroup analysis, the pooled result from the studies with $<1000$ cases showed a negative correlation between fruit intake and the likelihood of NAFLD $\left(I^{2}=0.0 \%, P_{\text {heterogeneity }}=0.437\right.$; $\mathrm{OR}=0.651 ; 95 \% \mathrm{CI} 0 \cdot 483,0 \cdot 878 ; P=0.005)$, whereas the results of all other subgroup analyses were consistent with the above overall analysis. Evidence of significant heterogeneity was still observed in the stratified analyses of the Asian studies $\left(I^{2}=74 \cdot 4 \%\right)$, the studies with $\geq 1000$ cases $\left(I^{2}=55 \cdot 2 \%\right)$ and the studies with validated dietary assessment $\left(I^{2}=72 \cdot 2 \%\right)$ (online Supplementary Table S6).

\section{Vegetables}

A total of eight studies assessed the effect of vegetable consumption on the likelihood of NAFLD, which include six cross-sectional studies (with 4523 cases) and two case-control studies (with 3074 cases) $)^{(14,15,34,36,38,44,45,47)}$. There was no significant association between vegetable intake and the likelihood of NAFLD among the cross-sectional studies $\left(I^{2}=50 \cdot 0 \%\right.$, 


\begin{tabular}{|c|c|c|}
\hline Study & & $\%$ \\
\hline ID & ES $(95 \% \mathrm{Cl})$ & Weight \\
\hline Chiu et al. (2018) & $1 \cdot 01(0.91,1 \cdot 12)$ & $33 \cdot 49$ \\
\hline Baratta et al. (2017) & $2 \cdot 18(0.99,4 \cdot 83)$ & 11.96 \\
\hline Shim et al. (2017) & $0.29(0.11,0.75)$ & $9 \cdot 19$ \\
\hline Chan et al. (2015) & $1.00(0.61,1.64)$ & $19 \cdot 95$ \\
\hline Zelber-Sagi et al. (2007) & $0.73(0.52,1.04)$ & $25 \cdot 42$ \\
\hline Overall $\left(I^{2}=69.4 \%, P=0.011\right)$ & $0.91(0 \cdot 65,1 \cdot 28)$ & $100 \cdot 00$ \\
\hline
\end{tabular}

Fig. 7. Random effects meta-analysis of cross-sectional studies that examined fish consumption and non-alcoholic fatty liver disease (NAFLD) risk. Weights are from random effects analysis. ES, effect size.

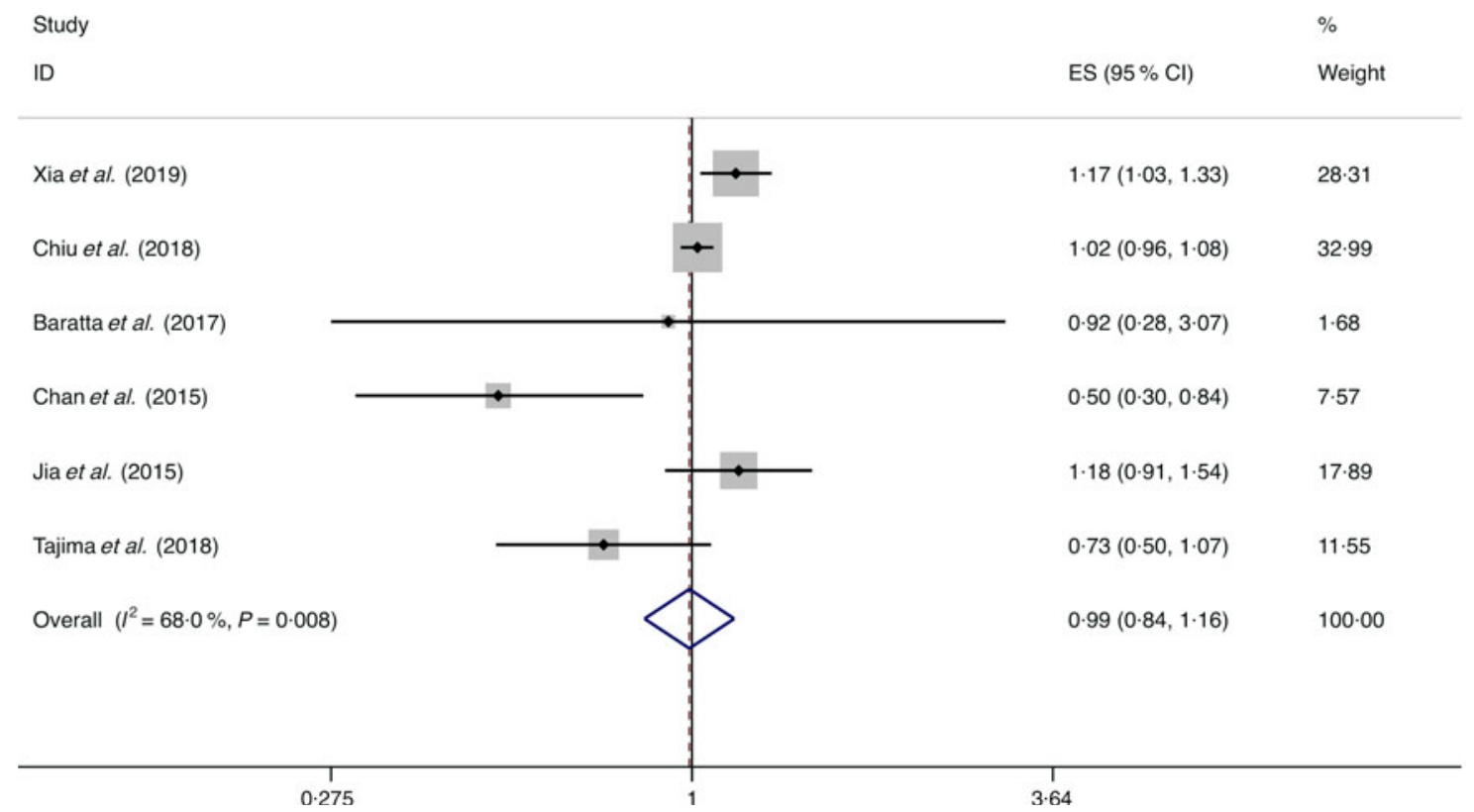

Fig. 8. Random effects meta-analysis of cross-sectional studies that examined fruit consumption and non-alcoholic fatty liver disease (NAFLD) risk. Weights are from random effects analysis. ES, effect size.

$\left.P_{\text {heterogeneity }}=0 \cdot 075 ; \mathrm{OR}=1 \cdot 005 ; 95 \% \mathrm{CI} 0 \cdot 976,1 \cdot 035 ; P=0 \cdot 725\right)$ and case-control studies $\left(I^{2}=0.0 \%, \quad P_{\text {heterogeneity }}=0 \cdot 884\right.$ $\mathrm{OR}=0.993 ; 95 \%$ CI $0.897,1 \cdot 1000 ; P=0.898)$ (Fig. 10$)$.

In subgroup analysis, the pooled result from the studies with $<1000$ cases showed a negative correlation between vegetables intake and the likelihood of NAFLD $\left(I^{2}=0.0 \%, P_{\text {heterogeneity }}=\right.$ $0.398 ; \mathrm{OR}=0.696 ; 95 \%$ CI $0.528,0.916 ; P=0.010)$, whereas the results of all other subgroup analyses were consistent with the above overall analysis. Evidence of significant heterogeneity was still found in the stratified analyses of the Asian studies
$\left(I^{2}=60.0 \%\right)$ and the studies with validated dietary assessment $\left(I^{2}=59.8 \%\right)$ (online Supplementary Table S7).

\section{Eggs}

A total of three studies assessed the effect of egg consumption on the likelihood of NAFLD, which include two cross-sectional studies (with 2131 cases) and one case-control study (with 169 cases $)^{(34,36,43)}$. Neither the cross-sectional studies $\left(I^{2}=0 \cdot 0 \%\right.$, $P_{\text {heterogeneity }}=0.532 ; \quad \mathrm{OR}=0.969 ; 95 \%$ CI $0.815,1.153$; 


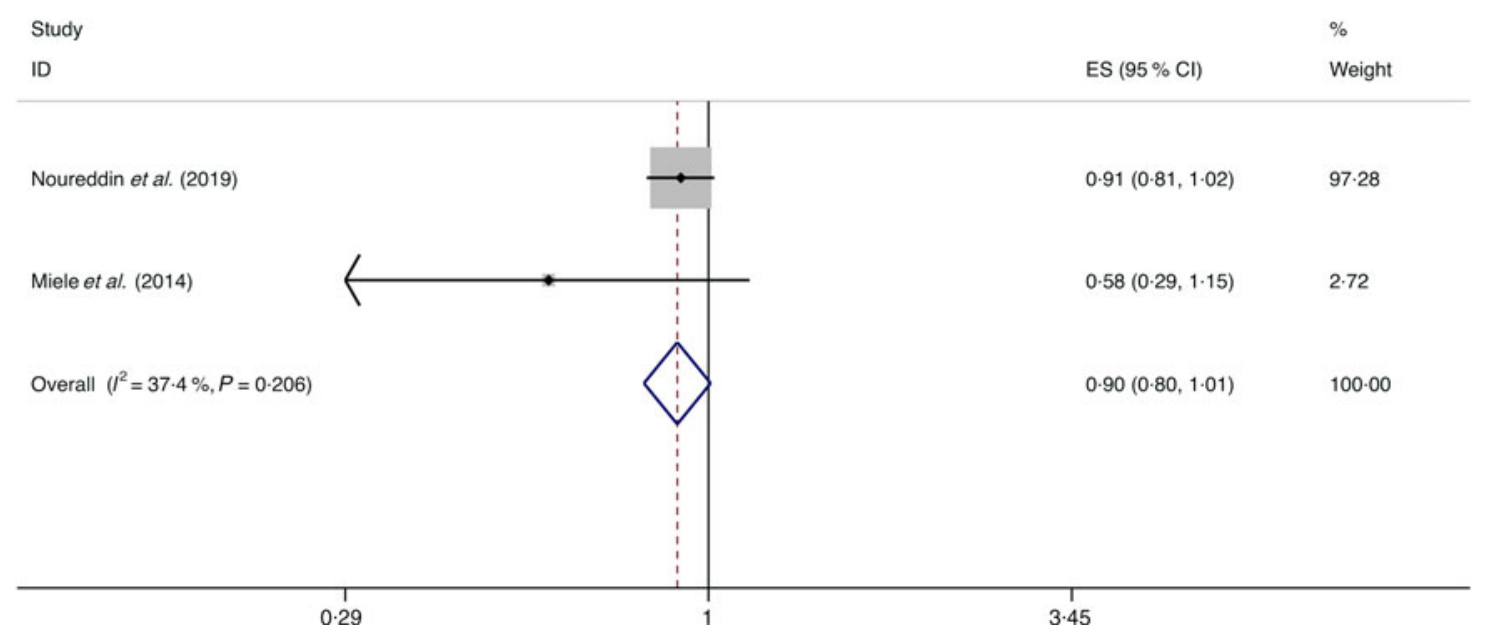

Fig. 9. Fixed effects meta-analysis of case-control studies that examined fruit consumption and non-alcoholic fatty liver disease (NAFLD) risk. Weights are from fixed effects analysis. ES, effect size.

\begin{tabular}{|c|c|c|}
\hline Study & & $\%$ \\
\hline ID & $\mathrm{ES}(95 \% \mathrm{Cl})$ & Weight \\
\hline Cross-sectional & & \\
\hline Chiu et al. (2018) & $1.01(0.98,1.04)$ & $90 \cdot 25$ \\
\hline Baratta et al. (2017) & $1.02(0.32,3.32)$ & 0.06 \\
\hline Shim et al. (2017) & $0.55(0.20,0.85)$ & 0.15 \\
\hline Chan et al. (2015) & $0.51(0.30,0.87)$ & 0.28 \\
\hline Jia et al. (2015) & $0.97(0.73,1.29)$ & 0.97 \\
\hline Tajima et al. male (2018) & $0.83(0.57,1.21)$ & 0.56 \\
\hline Subtotal $\left(l^{2}=50.0 \%, P=0.075\right)$ & $1.01(0.98,1.04)$ & $92 \cdot 27$ \\
\hline Case-control & & \\
\hline Katsagoni et al. (2016) & $1.01(0.79,1 \cdot 29)$ & $1 \cdot 33$ \\
\hline Noureddin et al. (2019) & $0.99(0 \cdot 88,1 \cdot 10)$ & $6 \cdot 40$ \\
\hline Subtotal $\left(I^{2}=0.0 \%, P=0.884\right)$ & $0.99(0.90,1 \cdot 10)$ & $7 \cdot 73$ \\
\hline Heterogeneity between groups: $P=0$ & & \\
\hline Overall $\left(I^{2}=30.5 \%, P=0.184\right)$ & $1.00(0.98,1.03)$ & $100 \cdot 00$ \\
\hline
\end{tabular}

Fig. 10. Fixed effects meta-analysis of prospective studies that examined vegetable consumption and non-alcoholic fatty liver disease (NAFLD) risk. Weights are from fixed effects analysis. ES, effect size.

$P=0 \cdot 722)$ nor the case-control study (OR $=0 \cdot 966 ; 95 \%$ CI $0 \cdot 453$, $2 \cdot 060 ; P=0 \cdot 929)$ showed the association between egg consumption and NAFLD (Fig. 11).

\section{Dairy products}

A total of four studies assessed the effect of dairy product consumption on the likelihood of NAFLD, which include three cross-sectional studies (with 6789 cases) and one case-control study (with 143 cases) $)^{(34,36,39,50)}$. Meta-analysis results from the three heterogeneous cross-sectional studies $\left(I^{2}=55.7 \%\right.$, $P=0 \cdot 105)$ showed no significant association between dairy product consumption and the likelihood of NAFLD $(\mathrm{OR}=0.954 ; \quad 95 \%$ CI 0.824, 1.104; $\quad P=0.524) \quad$ (Fig. 12). However, the result from the case-control study showed that dairy product consumption was positively associated with the possibility of NAFLD (OR $=1.192 ; 95 \%$ CI 1.002, 1.419; $P=0.048$ ). 


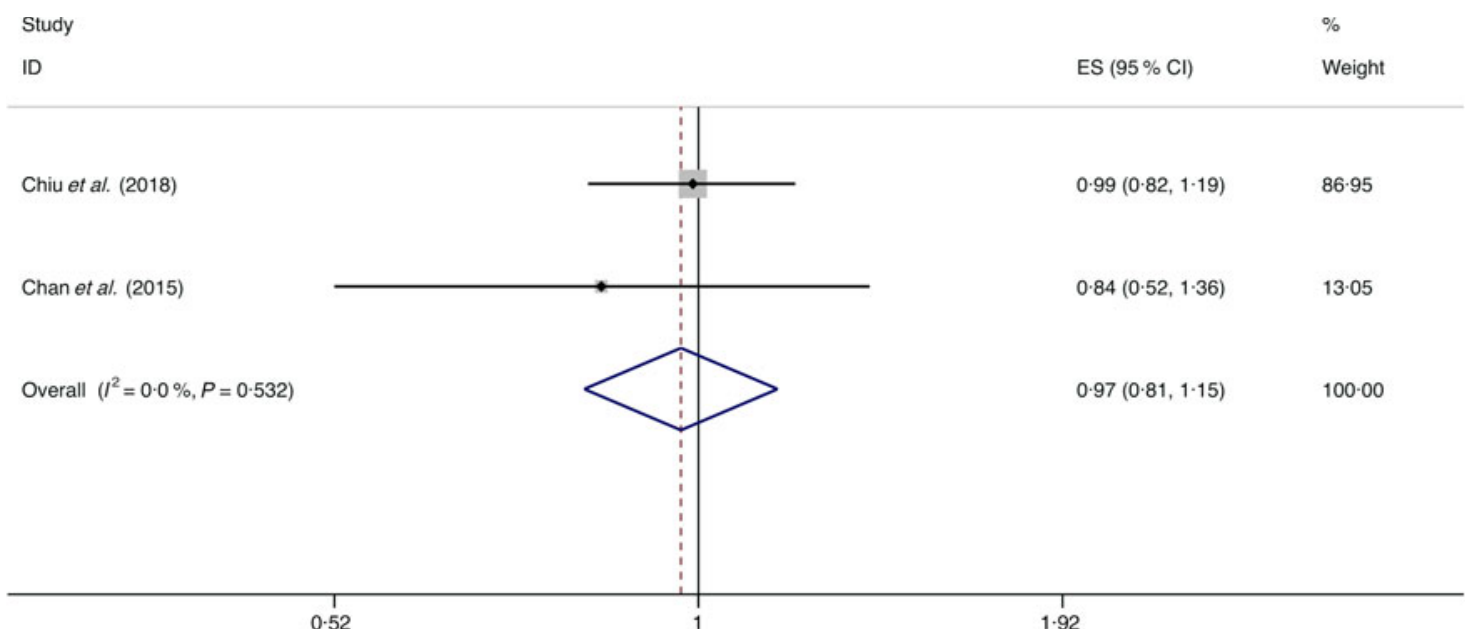

Fig. 11. Fixed effects meta-analysis of cross-sectional studies that examined egg consumption and non-alcoholic fatty liver disease (NAFLD) risk. Weights are from fixed effects analysis. ES, effect size.

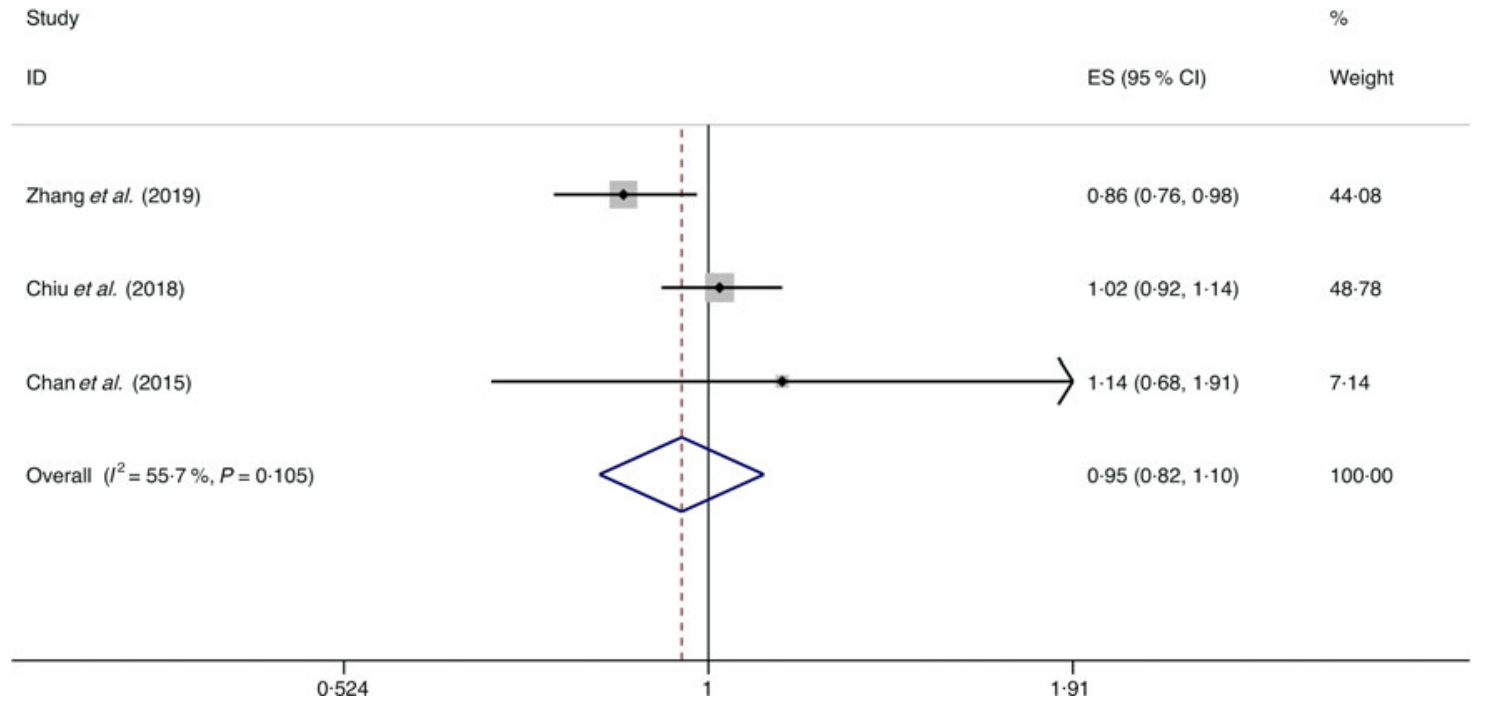

Fig. 12. Random effects meta-analysis of cross-sectional studies that examined dairy product consumption and non-alcoholic fatty liver disease (NAFLD) risk. Weights are from random effects analysis. ES, effect size.

\section{Legumes}

A total of four studies assessed the effect of legume consumption on the likelihood of NAFLD, which include three cross-sectional studies (with 2614 cases) and one case-control study (with 196 cases) $)^{(13,14,34,36)}$. Meta-analysis results from the three homogeneous cross-sectional studies $\left(I^{2}=0.0 \%\right.$, $P=0.507)$ showed no significant association between legume consumption and the likelihood of NAFLD (OR $=0.943 ; 95 \%$ CI $0.877,1.014 ; P=0.115$ ) (Fig. 13). However, the result from the case-control study showed legume consumption was negatively associated with the possibility of NAFLD (OR $=0.730$; $95 \%$ CI $0 \cdot 637,0 \cdot 836 ; P=0 \cdot 000)$.

\section{Sensitivity analyses}

In the sensitivity analyses, the findings from the studies with low risk of bias suggest a stronger positive association between red meat $\left(I^{2}=0 \cdot 0 \%, P_{\text {heterogeneity }}=0 \cdot 817 ;\right.$ OR $=1 \cdot 218 ; 95 \% \mathrm{CI} 1 \cdot 018$,
$1.458 ; P=0.031)$ and soft drinks $\left(I^{2}=0.0 \%, P_{\text {heterogeneity }}=0.880\right.$; $\mathrm{OR}=1.575 ; 95 \%$ CI $1 \cdot 133,2 \cdot 189 ; P=0 \cdot 007)$ intake and the possibility of NAFLD (online Supplementary Tables S4 and S5). Moreover, an inverse association was found by sensitivity analysis between vegetable consumption and NAFLD $\left(I^{2}=10 \cdot 2 \%\right.$, $P_{\text {heterogeneity }}=0.291 ; \quad$ OR $=0.574 ; \quad 95 \%$ CI $0.353,0.932$; $P=0.025$ ) in the studies with low risk of bias (online Supplementary Table S7).

\section{Discussion}

In this meta-analysis, the results revealed that intake of red meat and soft drinks was associated with an increase in the likelihood of NAFLD, whereas intake of nuts was negatively associated with the possibility of NAFLD. It is noteworthy that most foods included in the meta-analysis (whole grains, refined grains, fish, fruits, vegetables, eggs, dairy products and legumes) may have no significant effect on the likelihood of NAFLD. 


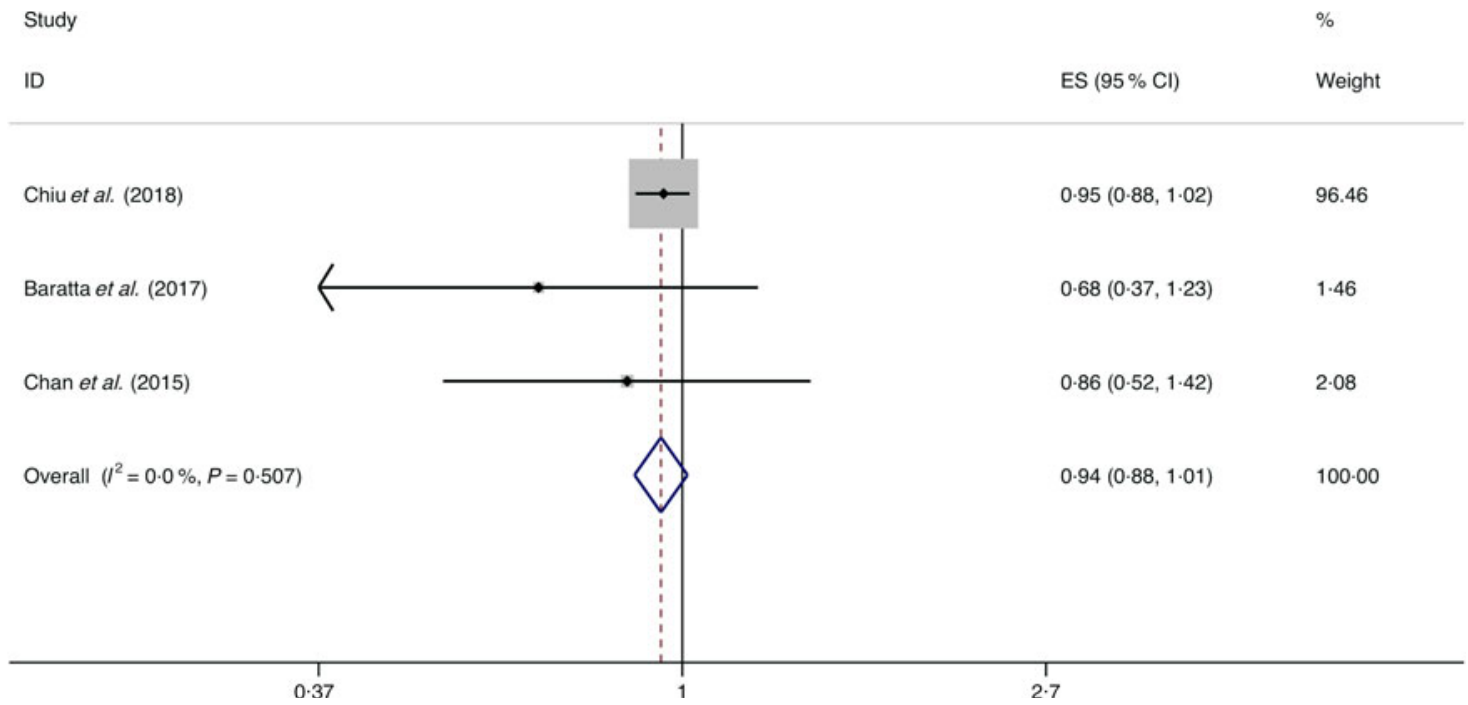

Fig. 13. Random effects meta-analysis of cross-sectional studies that examined legume consumption and non-alcoholic fatty liver disease (NAFLD) risk. Weights are from random effects analysis. ES, effect size.

To our knowledge, this is the first meta-analysis investigating the relationship between food groups (refined grains, whole grains, fish, red meat, vegetables, fruits, soft drinks, eggs, legumes, nuts and dairy products) and the likelihood of NAFLD. The pooled results of our meta-analysis are in accordance with other systematic reviews and meta-analyses, indicating that consumption of red meat and sugar- and artificially sweetened soda is positively associated with $\operatorname{NAFLD~}^{(53,54)}$. Firstly, red meat rich in saturated fat increases hepatic lipid accumulation and insulin resistance via reducing lipid oxidation and increasing lipid synthesis ${ }^{(55,56)}$. Additionally, haem Fe intake reduces insulin sensitivity through cellular oxidation stress ${ }^{(57)}$. Red meat is often processed with a lot of $\mathrm{Na}$ and preserved with nitrites, which is related to increase the likelihood of insulin resistance and NAFLD ${ }^{(58,59)}$. Secondly, several studies have also shown that higher consumption of soft drinks is associated with a greater likelihood of NAFLD and a series of metabolic syndromes $^{(60,61)}$. Soft drinks provide a large amount of sugar and excessive energy content, which led to rapidly increased insulin level and postprandial glucose ${ }^{(60,62)}$. Lebda et al. stated that longterm intake of soft drinks is prospectively associated with the level of ALT which represents liver inflammation ${ }^{(63)}$.

Consistent with other studies, our meta-analysis shows that nut consumption reduces the likelihood of NAFLD. A study with 12946 participants indicated that nut consumption was positively associated with healthier nutrition and lifestyle ${ }^{(64)}$; another study displayed that risk factors associated with NAFLD and CVD were improved after regular nut consumption ${ }^{(65)}$

However, the current research still fails to reach a consistent conclusion on the possibility of NAFLD with intake of fish, legumes, fruits, vegetables and dairy products. In the crosssectional studies, the pooled results showed that no significant associations were observed between consumption of both fish $(\mathrm{OR}=0.908)$ and legumes $(\mathrm{OR}=0.943)$ and the likelihood of NAFLD; but in the case-control studies, the results showed that consumption of both fish $(\mathrm{OR}=0.845)$ and legumes $(\mathrm{OR}=0.730)$ decreased the possibility of NAFLD. In the cross- sectional studies and the case-control studies, the pooled results showed no significant associations between intake of both fruits and vegetables and the likelihood of NAFLD; but in the crosssectional studies with $<1000$ cases, the pooled results showed that consumption of both fruits $(\mathrm{OR}=0.651)$ and vegetables (OR $=0.696$ ) significantly reduced the possibility of NAFLD. Moreover, in the cross-sectional studies with low risk of bias, the pooled results showed that intake of vegetables $(\mathrm{OR}=0.574)$ also reduced the possibility of NAFLD. On the contrary, in the cross-sectional studies, the pooled results showed that no significant association was observed between dairy product consumption and the likelihood of NAFLD, but in the casecontrol study, the result showed that dairy product consumption increased the possibility of NAFLD $(\mathrm{OR}=1 \cdot 192)^{(50)}$. Taken together, the above findings suggest that higher consumption of fish, legumes, fruits and vegetables appeared to have a protective trend against NAFLD likelihood, while intake of more milk may be an adverse effect on the likelihood of NAFLD.

$n$-3 PUFA in fish and isoflavones in legumes have been shown to reduce lipid accumulation and liver enzyme levels, to improve insulin sensitivity and to have anti-inflammatory effects and thus are associated with the prevention of the development of hepatic steatosis, NAFLD, non-alcoholic steatohepatitis and fibrosis ${ }^{(66-68)}$. Fruits and vegetables are rich in fibre, antioxidants such as polyphenols, which help prevent the occurrence of NAFLD. In addition to the antioxidant effect, polyphenols also have beneficial effects on metabolic homoeostasis in vivo and in vitro NAFLD model, with anti-inflammatory and anti-fibrosis effects. In general, they inhibit de novo fat synthesis and stimulate $\beta$-oxidation in the liver ${ }^{(69)}$. On the other hand, the case-control study ${ }^{(50)}$ as mentioned above found that dairy products (mainly referring to cheese) increased the likelihood of NAFLD. This may be related to the fact that cheese contains more SFA that increase liver steatosis ${ }^{(39)}$. More studies are needed to further confirm the relationship between fish, legumes, fruits, vegetables and dairy products and the likelihood of NAFLD. 
In recent years, on the basis of the components of the Mediterranean diet, the literature reports its beneficial effects in preventing major chronic diseases, including obesity, diabetes, CVD and some forms of cancers ${ }^{(70-73)}$. More importantly, a growing body of evidence has supported the idea that the Mediterranean diet, associated with exercise and cognitive behaviour therapy, may be the reference nutritional profile for the prevention and the treatment of NAFLD patients ${ }^{(74-76)}$. It is characterised by an abundance of consumption of whole grains, vegetables, fruits, legumes, nuts and olive oil (rich in monounsaturated fat); a moderate intake of fish and poultry; low consumption of red/processed meat and dairy products and low to moderate consumption of alcohol during meals ${ }^{(75)}$. From our meta-analysis results, a high to moderate intake of nuts, legumes, fish, eggs, whole grains and vegetables, and low consumption of red meat and soft drinks should be recommended for patients with NAFLD, which is roughly similar to the diet composition of the Mediterranean diet. At present, the Mediterranean diet is the latest recommended dietary pattern for NAFLD patients in EASL-EASD-EASO clinical practice guidelines ${ }^{(6)}$.

Most of the studies measured food intake with a FFQ, which is easy for the administration to assess long-term habitual food consumption. It contains numerous food items with specific serving sizes and frequency categories and has the following advantages: first, since the questionnaire is structured, it is convenient and simple; second, the implementation is simple and easy to understand, and the participation rate of subjects is high; third, it can be used to analyse the correlation between specific nutrients or related foods and diseases. However, there are some limitations. First, it may cause bias due to errors in the estimation of portion sizes; second, cooking methods and seasoning dosage are not easy to estimate; there may be a large error range. Although the debate about the utility of FFQ in nutritional epidemiological studies is often polarised, dietary data derived from it have proven useful in addressing important research questions $^{(77)}$. Of the twenty-four studies included in our metaanalysis, twenty-one studies used FFQ to investigate dietary intake. Since the implementation is simple and the content design is easy to understand, subjects will be more willing to cooperate, thus guaranteeing the reliability of the results of this meta-analysis.

Some strengths of this meta-analysis are as follow: (1) we investigated a variety of foods; (2) all the studies included in our meta-analysis were low to moderate bias risk; (3) all the studies included in our meta-analysis used multiple logistic regressions to reduce the effect of confounders on the correlation of NAFLD with food consumption. However, several limitations of this meta-analysis should be noted. First, because the overall number of studies included was small, subgroup and sensitivity analyses were limited to four of the eleven food groups (red meat, soft drinks, fruits and vegetables). Consistent with this, publication bias could not be tested as the overall low number of included studies. Also, the results of several food groups may be biased due to too few studies included (eggs $=$ three studies, dairy products $=$ four studies, legumes $=$ four studies, whole grains $=$ three studies). Lastly, since most studies do not stratify food intake, it is not possible to perform linear or nonlinear dose-response of different food groups. Also, the included studies used different units or different standards to measure food groups.

In summary, this meta-analysis with twenty-four studies identified eleven food groups associating the likelihood of NAFLD. The results were broadly consistent with the current dietary recommendation for the management of NAFLD. Larger and more precise studies are required to further assess the association and the underlying mechanisms between food groups and the possibility of NAFLD.

\section{Acknowledgements}

The authors' contributions were as follows - all authors designed the research (project conception, development of the overall research plan) and approved the final manuscript; K. H. and Y. L. designed and conducted the research (conducted the systematic search, screened the literature and extracted the data); K. H. completed the first draft of the manuscript; X. G. and L. Z. analysed the data and performed the statistical analyses; S. T. had primary responsibility for final content; L. Z. critically reviewed the manuscript. None of the other authors reported a conflict of interest related to the study.

\section{Supplementary material}

For supplementary material referred to in this article, please visit https://doi.org/10.1017/S0007114520000914

\section{References}

1. Younossi ZM, Koenig AB, Abdelatif D, et al. (2016) Global epidemiology of nonalcoholic fatty liver disease-meta-analytic assessment of prevalence, incidence, and outcomes. Hepatology 64, 73-84.

2. Friedman SL, Neuschwander-Tetri BA, Rinella M, et al. (2018) Mechanisms of NAFLD development and therapeutic strategies. Nat Med 24, 908-922.

3. Singh S, Allen AM, Wang Z, et al. (2015) Fibrosis progression in nonalcoholic fatty liver $v$. nonalcoholic steatohepatitis: a systematic review and meta-analysis of paired-biopsy studies. Clin Gastroenterol Hepatol 13, 643-654. e1-9; quiz e39-40.

4. Kitade H, Chen G, Ni Y, et al. (2017) Nonalcoholic fatty liver disease and insulin resistance: new insights and potential new treatments. Nutrients $\mathbf{9}, \mathrm{E} 387$.

5. Abenavoli L, Boccuto L, Federico A, et al. (2019) Diet and non-alcoholic fatty liver disease: the Mediterranean way. Int J Environ Res Public Health 16, 3011.

6. European Association for the Study of the Liver (EASL), European Association for the Study of Diabetes (EASD) \& European Association for the Study of Obesity (EASO) (2016) EASL-EASD-EASO clinical practice guidelines for the management of non-alcoholic fatty liver disease. $J$ Hepatol 64, 1388-1402.

7. Abenavoli L, Di Renzo L, Boccuto L, et al. (2018) Health benefits of Mediterranean diet in nonalcoholic fatty liver disease. Expert Rev Gastroenterol Hepatol 12, 873-881.

8. Loria P, Adinolfi LE, Bellentani S, et al. (2010) Practice guidelines for the diagnosis and management of nonalcoholic fatty liver disease. A decalogue from the Italian Association for the Study of the Liver (AISF) expert committee. Dig Liver Dis $\mathbf{4 2}$, 272-282. 
9. Asrih M \& Jornayvaz FR (2014) Diets and nonalcoholic fatty liver disease: the good and the bad. Clin Nutr 33, 186-190.

10. Dixon JB, Bhathal PS, Hughes NR, et al. (2004) Nonalcoholic fatty liver disease: improvement in liver histological analysis with weight loss. Hepatology 39, 1647-1654.

11. Carvalhana S, Machado MV \& Cortez-Pinto H (2012) Improving dietary patterns in patients with nonalcoholic fatty liver disease. Curr Opin Clin Nutr Metab Care 15, 468-473.

12. Abid A, Taha O, Nseir W, et al. (2009) Soft drink consumption is associated with fatty liver disease independent of metabolic syndrome. J Hepatol 51, 918-924.

13. Bahrami A, Teymoori F, Eslamparast T, et al. (2019) Legume intake and risk of nonalcoholic fatty liver disease. Indian J Gastroenterol 38, 55-60.

14. Baratta F, Pastori D, Polimeni L, et al. (2017) Adherence to Mediterranean diet and non-alcoholic fatty liver disease: effect on insulin resistance. Am J Gastroenterol 112, 1832-1839.

15. Katsagoni CN, Georgoulis M, Papatheodoridis GV, et al. (2017) Associations between lifestyle characteristics and the presence of nonalcoholic fatty liver disease: a case-control study. Metab Syndr Relat Disord 15, 72-79.

16. Rietman A, Sluik D, Feskens EJM, et al. (2018) Associations between dietary factors and markers of NAFLD in a general Dutch adult population. Eur J Clin Nutr 72, 117-123.

17. Shi L, Liu ZW, Li Y, et al. (2012) The prevalence of nonalcoholic fatty liver disease and its association with lifestyle/dietary habits among university faculty and staff in Chengdu. Biomed Environ Sci 25, 383-391.

18. Shim P, Choi D \& Park Y (2017) Association of blood fatty acid composition and dietary pattern with the risk of non-alcoholic fatty liver disease in patients who underwent cholecystectomy. Ann Nutr Metab 70, 303-311.

19. Buttriss JL (2016) The Eatwell Guide refreshed. Nutr Bull. 41 135-141

20. Marshall TA (2011) Dietary guidelines for Americans, 2010: an update. J Am Dent Assoc 142, 654-656.

21. Stroup DF, Berlin JA, Morton SC, et al. (2000) Meta-analysis of observational studies in epidemiology: a proposal for reporting. Meta-analysis of Observational Studies in Epidemiology (MOOSE) group. JAMA 283, 2008-2012.

22. Moher D, Shamseer L, Clarke M, et al. (2015) Preferred reporting items for systematic review and meta-analysis protocols (PRISMA-P) 2015 statement. Syst Rev 4, 1.

23. Cumpston M, Li T, Page MJ, et al. (2019) Updated guidance for trusted systematic reviews: a new edition of the Cochrane Handbook for systematic reviews of interventions. Cochrane Database Syst Rev, issue 10, ED000142.

24. Kleiner DE, Brunt EM, Van Natta M, et al. (2005) Design and validation of a histological scoring system for nonalcoholic fatty liver disease. Hepatology 41, 1313-1321.

25. Speliotes EK, Massaro JM, Hoffmann U, et al. (2010) Fatty liver is associated with dyslipidemia and dysglycemia independent of visceral fat: the Framingham Heart Study. Hepatology $\mathbf{5 1}$, 1979-8197.

26. Singh D, Das CJ \& Baruah MP (2013) Imaging of non alcoholic fatty liver disease: a road less travelled. Indian J Endocrinol Metab 17, 990-995.

27. Iwasaki M, Takada Y, Hayashi M, et al. (2004) Noninvasive evaluation of graft steatosis in living donor liver transplantation. Transplantation 78, 1501-1505.

28. Browning JD, Szczepaniak LS, Dobbins R, et al. (2004) Prevalence of hepatic steatosis in an urban population in the United States: impact of ethnicity. Hepatology 40, 1387-1395.

29. Sterne JA, Hernan MA, Reeves BC, et al. (2016) ROBINS-I: a tool for assessing risk of bias in non-randomised studies of interventions. BMJ 355, i4919.
30. DerSimonian R \& Laird N (1986) Meta-analysis in clinical trials. Control Clin Trials 7, 177-188.

31. Higgins JP \& Thompson SG (2002) Quantifying heterogeneity in a meta-analysis. Stat Med 21, 1539-1558.

32. Egger M, Davey Smith G, Schneider M, et al. (1997) Bias in meta-analysis detected by a simple, graphical test. $B M J \mathbf{3 1 5}$, 629-634.

33. Higgins JP \& Green SE (2011) Cochrane Handbook for Systematic Reviews of Interventions version 5.1.0. Oxford: The Cochrane Collaboration. http://handbook-5-1.cochrane.org/

34. Chan R, Wong VW, Chu WC, et al. (2015) Diet-quality scores and prevalence of nonalcoholic fatty liver disease: a population study using proton-magnetic resonance spectroscopy. PLOS ONE 10, e0139310.

35. Chen BB, Han Y, Pan X, et al. (2019) Association between nut intake and non-alcoholic fatty liver disease risk: a retrospective case-control study in a sample of Chinese Han adults. BMJ Open 9, e028961.

36. Chiu TH, Lin MN, Pan WH, et al. (2018) Vegetarian diet, food substitution, and nonalcoholic fatty liver. Tzu Chi Med J 30, 102-109.

37. Georgoulis M, Kontogianni MD, Tileli N, et al. (2014) The impact of cereal grain consumption on the development and severity of non-alcoholic fatty liver disease. Eur J Nutr $\mathbf{5 3}$, $1727-1735$.

38. Jia Q, Xia Y, Zhang Q, et al. (2015) Dietary patterns are associated with prevalence of fatty liver disease in adults. Eur J Clin Nutr 69, 914-921.

39. Kalafati IP, Dimitriou M, Borsa D, et al. (2019) Fish intake interacts with TM6SF2 gene variant to affect NAFLD risk: results of a case-control study. Eur J Nutr 58, 1463-1473.

40. Ma J, Fox CS, Jacques PF, et al. (2015) Sugar-sweetened beverage, diet soda, and fatty liver disease in the Framingham Heart Study cohorts. J Hepatol 63, 462-469.

41. Meng G, Zhang B, Yu F, et al. (2018) Soft drinks consumption is associated with nonalcoholic fatty liver disease independent of metabolic syndrome in Chinese population. Eur J Nutr 57, 2113-2121.

42. Miele L, Dall'Armi V, Cefalo C, et al. (2014) A case-control study on the effect of metabolic gene polymorphisms, nutrition, and their interaction on the risk of non-alcoholic fatty liver disease. Genes Nutr 9, 383.

43. Mokhtari Z, Poustchi H, Eslamparast T, et al. (2017) Egg consumption and risk of non-alcoholic fatty liver disease. World J Hepatol 9, 503-509.

44. Noureddin M, Zelber-Sagi S, Wilkens LR, et al. (2019) Diet associations with nonalcoholic fatty liver disease in an ethnically diverse population: the Multiethnic Cohort. Hepatology (epublication ahead of print version 25 September 2019).

45. Tajima R, Kimura T, Enomoto A, et al. (2019) No association between fruits or vegetables and non-alcoholic fatty liver disease in middle-aged men and women. Nutrition 61, 119-124.

46. Tajima R, Kimura T, Enomoto A, et al. (2017) Association between rice, bread, and noodle intake and the prevalence of non-alcoholic fatty liver disease in Japanese middle-aged men and women. Clin Nutr 36, 1601-1608.

47. Xia Y, Lu Z, Lu M, et al. (2019) Raw orange intake is associated with higher prevalence of non-alcoholic fatty liver disease in an adult population. Nutrition 60, 252-260.

48. Zelber-Sagi S, Ivancovsky-Wajcman D, Fliss Isakov $\mathrm{N}$, et al. (2018) High red and processed meat consumption is associated with non-alcoholic fatty liver disease and insulin resistance. J Hepatol 68, 1239-1246.

49. Zelber-Sagi S, Nitzan-Kaluski D, Goldsmith R, et al. (2007) Long term nutritional intake and the risk for non-alcoholic fatty liver disease (NAFLD): a population based study. J Hepatol 47, 711-717. 
50. Zhang S, Fu J, Zhang Q, et al. (2019) Association between habitual yogurt consumption and newly diagnosed nonalcoholic fatty liver disease. Eur J Clin Nutr 74, 491-499.

51. Zhang S, Fu J, Zhang Q, et al. (2019) Association between nut consumption and non-alcoholic fatty liver disease in adults. Liver Int 39, 1732-1741.

52. Zhou X, Li Y, Zhang X, et al. (2019) Independent markers of nonalcoholic fatty liver disease in a gentrifying population-based Chinese cohort. Diabetes Metab Res Rev 35 e3156.

53. Wijarnpreecha K, Thongprayoon C, Edmonds PJ, et al. (2016) Associations of sugar- and artificially sweetened soda with nonalcoholic fatty liver disease: a systematic review and metaanalysis. QJM 109, 461-466.

54. Mirmiran P, Amirhamidi Z, Ejtahed HS, et al. (2017) Relationship between diet and Non-alcoholic fatty liver disease: a review article. Iran J Public Health 46, 1007-1017.

55. de Meijer VE, Le HD, Meisel JA, et al. (2010) Dietary fat intake promotes the development of hepatic steatosis independently from excess caloric consumption in a murine model. Metabolism 59, 1092-1105.

56. Hernandez EA, Kahl S, Seelig A, et al. (2017) Acute dietary fat intake initiates alterations in energy metabolism and insulin resistance. J Clin Invest 127, 695-708.

57. Zhao Z, Li S, Liu G, et al. (2012) Body iron stores and heme-iron intake in relation to risk of type 2 diabetes: a systematic review and meta-analysis. PLOS ONE 7, e41641.

58. Kim Y, Keogh J \& Clifton P (2015) A review of potential metabolic etiologies of the observed association between red meat consumption and development of type 2 diabetes mellitus. Metabolism 64, 768-779.

59. Choi Y, Lee JE, Chang Y, et al. (2016) Dietary sodium and potassium intake in relation to non-alcoholic fatty liver disease. Br J Nutr 116, 1447-1456.

60. Assy N, Nasser G, Kamayse I, et al. (2008) Soft drink consumption linked with fatty liver in the absence of traditional risk factors. Can J Gastroenterol 22, 811-816.

61. Siddiqi Z, Karoli R, Fatima J, et al. (2017) Soft drinks consumption and the risk of nonalcoholic fatty liver disease. $J$ Assoc Physicians India 65, 28-32.

62. Dekker MJ, Su Q, Baker C, et al. (2010) Fructose: a highly lipogenic nutrient implicated in insulin resistance, hepatic steatosis, and the metabolic syndrome. Am J Physiol Endocrinol Metab 299, E685-E694.

63. Lebda MA, Tohamy HG \& El-Sayed YS (2017) Long-term soft drink and aspartame intake induces hepatic damage via dysregulation of adipocytokines and alteration of the lipid profile and antioxidant status. Nutr Res $\mathbf{4 1}, 47-55$.
64. Witkowska AM, Waskiewicz A, Zujko ME, et al. (2019) The consumption of nuts is associated with better dietary and lifestyle patterns in polish adults: results of WOBASZ and WOBASZ II surveys. Nutrients 11, 1410.

65. Coates AM, Hill AM \& Tan SY (2018) Nuts and cardiovascular disease prevention. Curr Atheroscler Rep 20, 48.

66. Bouzianas DG, Bouziana SD \& Hatzitolios AI (2013) Potential treatment of human nonalcoholic fatty liver disease with long-chain omega-3 polyunsaturated fatty acids. Nutr Rev $\mathbf{7 1}$, 753-771.

67. Liu H, Zhong H, Leng L, et al. (2017) Effects of soy isoflavone on hepatic steatosis in high fat-induced rats. J Clin Biochem Nutr 61, 85-90.

68. Masterton GS, Plevris JN \& Hayes PC (2010) Review article: omega-3 fatty acids - a promising novel therapy for nonalcoholic fatty liver disease. Aliment Pharmacol Ther 31, 679-692.

69. Salomone F, Godos J \& Zelber-Sagi S (2016) Natural antioxidants for non-alcoholic fatty liver disease: molecular targets and clinical perspectives. Liver Int 36, 5-20.

70. Benetou V, Trichopoulou A, Orfanos P, et al. (2008) Conformity to traditional Mediterranean diet and cancer incidence: the Greek EPIC cohort. Br J Cancer 99, 191-195.

71. Beunza JJ, Toledo E, Hu FB, et al. (2010) Adherence to the Mediterranean diet, long-term weight change, and incident overweight or obesity: the Seguimiento Universidad de Navarra (SUN) cohort. Am J Clin Nutr 92, 1484-1493.

72. Estruch R, Ros E, Salas-Salvado J, et al. (2013) Primary prevention of cardiovascular disease with a Mediterranean diet. NEngl I Med 368, 1279-1290.

73. Salas-Salvado J, Bullo M, Estruch R, et al. (2014) Prevention of diabetes with Mediterranean diets: a subgroup analysis of a randomized trial. Ann Intern Med 160, 1-10.

74. Malakou E, Linardakis M, Armstrong MEG, et al. (2018) The combined effect of promoting the Mediterranean diet and physical activity on metabolic risk factors in adults: a systematic review and meta-analysis of randomised controlled trials. Nutrients 10, 1577.

75. Romero-Gomez M, Zelber-Sagi S \& Trenell M (2017) Treatment of NAFLD with diet, physical activity and exercise. J Hepatol $\mathbf{6 7}$, 829-846.

76. Trovato FM, Catalano D, Martines GF, et al. (2015) Mediterranean diet and non-alcoholic fatty liver disease: the need of extended and comprehensive interventions. Clin Nutr 34, 86-88.

77. Hebert JR, Hurley TG, Steck SE, et al. (2014) Considering the value of dietary assessment data in informing nutrition-related health policy. Adv Nutr 5, 447-455. 